\title{
Bombas de infusión continua de jeringa en unidades neonatales
}

\author{
NADIA SCHMIDT S. ${ }^{1}$, CLAUDIA SÁEZ H. ${ }^{2}$, ANDRÉS MATURANA P. ${ }^{3}$ \\ 1. Universidad de Chile. Santiago. Chile. \\ 2. Clínica Alemana de Santiago. Chile. \\ 3. Universidad del Desarrollo. Santiago. Chile; Clínica Alemana de Santiago. Chile.
}

\begin{abstract}
Continuous infusion pumps at neonatal intensive care units

Introduction: Continuous Infusion pumps (CIP) are frequently used at neonatal intensive care units (NICUs), to administer medications at flow rates lower than $1 \mathrm{ml} / \mathrm{H}$. Various factors have been shown to alter their performance. No studies have been completed in Chile in this area. Objectives: To describe frequent clinical practices to administer medications through CIP at NICUs in Chile, and suggest recommendations about it. Methods: Four professionals at NICUs in 19 centers were surveyed regarding syringe size, flow rate and precautions. Results: When starting countinuous infusion, $81.9 \%$ of surveyed professionals use 50 cc syringes, and $66.7 \%$ of them use flow rates under $1 \mathrm{ml} / \mathrm{h}$. Main precautions include elimination of bubbles in the circuit (51.4\%), review of conections (38.9\%) and precise fit between the syringe and the CIP (36.1\%). Conclusions: In surveyed NICUs, low flow rates and 50cc syringes are frequently used to infuse medications. NICU staff must consider factors that affect the performance of CIP, prefer smaller syringes and flow rate over $1 \mathrm{ml} / \mathrm{h} \mathrm{for}$ continuous infusion of drugs.
\end{abstract}

(Key words: Infusion pumps, syringes, intravenous infusions, low flow rates, intensive care units, newborn). Rev Chil Pediatr 2011; 82 (2): 123-128

\section{RESUMEN}

Introducción: En las unidades de cuidado intensivo neonatal (UCIN) se utilizan frecuentemente bombas de infusión continua (BIC) a flujos menores de $1 \mathrm{ml} / \mathrm{h}$ para administrar medicamentos. Está demostrado que distintos factores alteran el funcionamiento de las BIC a flujos bajos. En Chile, no existen estudios que describan prácticas relacionadas al uso de BIC. Objetivo: Describir prácticas clínicas frecuentes al administrar medicamentos en BIC en UCIN de Chile y proponer recomendaciones respecto a su uso. Método: Se encuestaron 4 profesionales de UCIN en 19 centros. Los principales aspectos evaluados fueron: tamaño de jeringa usado en las BIC, flujos de infusión continua utilizados y precauciones al instalar una BIC. Resultados: Al instalar una infusión continua, el 81,9\% de los profesionales encuestados usa jeringas de $50 \mathrm{ml}$ y el 66,7\% utiliza flujos me-

Trabajo recibido el 20 de julio de 2010, devuelto para corregir el 10 de noviembre de 2010, segunda versión el 02 de febrero de 2011, aceptado para publicación el 21 de febrero de 2011.

Correspondencia a:

Dr. Andrés Maturana P.

E-mail: andres.maturana.p@gmail.com 
nores de $1 \mathrm{ml} / \mathrm{h}$. Las principales precauciones fueron la eliminación de burbujas del circuito (51,4\%), la revisión de las conexiones del sistema (38,9\%) y el ajuste preciso entre jeringa y BIC (36,1\%). Conclusiones: En las UCIN estudiadas se utilizan frecuentemente flujos bajos y jeringas de $50 \mathrm{ml}$ para infundir medicamentos. Las UCIN deben considerar los factores que alteran el funcionamiento de las BIC y preferir el uso de jeringas de menor tamaño y flujos mayores de $1 \mathrm{ml} / \mathrm{h}$ para la infusión continua de medicamentos.

(Palabras clave: Bombas de infusión, jeringas, infusiones endovenosas, flujos bajos, unidades de cuidado intensivo, neonatos).

Rev Chil Pediatr 2011; 82 (2): 123-128

\section{Introducción}

La administración de medicamentos en infusión continua a flujos bajos (menores de $1 \mathrm{ml} / \mathrm{h}$ ) utilizando bombas de infusión continua de jeringa (BIC) es una práctica habitual en las unidades de cuidado intensivo neonatal (UCIN). Los recién nacidos en estado crítico muchas veces requieren la administración de múltiples medicamentos simultaneamente en infusión continua tales como inótropos, analgésicos y sedantes por lo que es necesario utilizar flujos bajos de infusión para evitar un aporte excesivo de volumen. El efecto de estos medicamentos depende de la llegada rápida y fiable de la infusión al paciente, por lo que es fundamental contar con sistemas de infusión exactos y confiables ${ }^{1,2}$.

Actualmente, se tiene conocimiento de distintos factores que afectan el adecuado funcionamiento de las BIC y que son amplificados al utilizar flujos bajos². Factores mecánicos, físicos y dinámicos comprometen tanto la precisión de la BIC, así como también el tiempo que la BIC demora en entregar efectivamente un medicamento al paciente. Algunos de los elementos que alteran su funcionamiento son el tamaño y tipo de jeringa utilizada ${ }^{3,4}$, el tipo de línea de infusión usada ${ }^{5,6}$, el ajuste mecánico entre la jeringa y la $\mathrm{BIC}^{7,8}$, la presencia de burbujas en el circuito de infusión ${ }^{9,10}$ y los cambios de posición en el eje vertical de la BIC durante la infusión ${ }^{11,12}$.

Un fenómeno importante de destacar y que es inherente al funcionamiento de las BIC es la existencia de un retraso desde el momento en que se pone la BIC en funcionamiento para iniciar la infusión, hasta que efectivamente comienza la entrega del medicamento al paciente $^{7}$. Varias investigaciones han demostrado que este retraso aumenta significativamente a flujos menores de $1 \mathrm{ml} / \mathrm{h}$ y al utilizar jeringas de mayor tamaño. Así se ha observado, que al utilizar jeringas de $50 \mathrm{ml}$ y flujos menores de $1 \mathrm{ml} / \mathrm{h}$ en una infusión continua, pueden pasar hasta 60 minutos antes del inicio real de la infusión al paciente ${ }^{13}$. Entre las medidas recomendadas para minimizar este problema destacan la administración de un bolo previo a la infusión ${ }^{7}$, el mayor cuidado en el posicionamiento de la jeringa evitando espacio libre entre el embolo y la $\mathrm{BIC}^{7,8}$, la eliminación de las burbujas del circuito ${ }^{9,10}$ y evitar los cambios de posición en el eje vertical de la BIC durante la infusión ${ }^{11,12}$.

Todos estos aspectos resultan relevantes en la práctica neonatal, especialmente si se considera que el uso de flujos bajos es indispensable. Actualmente, en nuestro medio no existen estudios que cuantifiquen el uso de flujos bajos, describan el tamaño de jeringas utilizadas para la infusión continua de medicamentos o que hayan investigado el uso de las medidas recomendadas para optimizar el tiempo de entrega real de la infusión al paciente. La revisión de estas prácticas clínicas en relación al uso de BIC resulta indispensable cuando se consideran todos los factores descritos en la literatura que pueden impactar la administración efectiva de medicamentos al utilizar flujos bajos.

El objetivo de este estudio es describir las prácticas clínicas más frecuentes en relación al uso de BIC para la administración de medicamentos en diferentes UCIN de Chile y hacer una propuesta de recomendaciones clínicas en esta área. 


\section{Pacientes y Método}

Se solicitó la aprobación del médico jefe y de la matrona o enfermera jefe de cada unidad de neonatología de 19 centros públicos y privados del país para su participación en este estudio.

Se realizó una encuesta a 1 matrona o enfermera de UCIN de turnos diferentes de cada uno de los centros estudiados, intentando $\mathrm{Cu}-$ brir al máximo la variabilidad de prácticas dentro de cada centro. La encuesta fue anónima y fue respondida individualmente por cada profesional, no existiendo consenso entre los distintos turnos.

Previo a su aplicación se realizó un estudio piloto que comprobó su reproducibilidad.

La encuesta incluyó 13 preguntas de selección múltiple y 1 pregunta de respuesta abierta. En las preguntas de selección múltiple los encuestados podían responder más de una alternativa. Estas preguntas consideraron los siguientes aspectos: tamaños de las jeringas utilizadas para la administración continua de medicamentos, tamaños de las jeringas usadas para la administración de medicamentos en BIC pero no en infusión continua, flujos preferentemente utilizados para la infusión continua de medicamentos; cantidad de aire o burbujas aceptadas en un circuito de infusión continua, considerando jeringa, conexiones y línea de infusión; y la realización o no de un bolo previo a la administración de un medicamento en infusión continua.

La pregunta de desarrollo investigó las precauciones que toman los profesionales al instalar una infusión continua de medicamento desde la colocación de la jeringa en la BIC hasta la puesta en marcha de ésta. Esta pregunta podía ser desarrollada en extenso por el profesional.

La información fue analizada manteniendo anónimo el centro estudiado y el profesional encuestado. Se realizó un análisis descriptivo con frecuencias en forma independiente para cada respuesta utilizando como unidad de análisis el profesional encuestado. Para el análisis estadistico se utilizo SPSS 12.0 para Windows, 2001. Chicago: SPSS Inc. Los resultados se expresaron en modo descriptivo como porcentajes. Debido a que cada pregunta de selección múltiple podía tener más de una respuesta los resultados no suman $100 \%$.

\section{Resultados}

Se estudiaron 19 unidades de neonatología en distintos centros públicos y privados de salud de Chile. En 17 de los centros incluidos la encuesta fue respondida individualmente por 4 profesionales de turnos diferentes. En una de las instituciones estudiadas, la encuesta fue respondida por un solo profesional con el consenso de sus pares. En otra sólo se logró encuestar a 3 profesionales de turnos distintos. De esta forma, la encuesta fue respondida por un total de 72 profesionales en representación de 19 centros.

Los profesionales encuestados, en su gran mayoria (81,9\%), utilizan jeringas de $50 \mathrm{ml}$ para la infusión continua de medicamentos (figura 1). Es interesante destacar que sólo los encuestados de dos centros no utilizan esta práctica clínica, favoreciendo el uso de jeringas de $20 \mathrm{ml}$ y $5 \mathrm{ml}$.

El 66,7\% (48 de 72 encuestados) de los profesionales utiliza flujos menores de $1 \mathrm{ml} / \mathrm{h}$ para la administración continua de medicamentos. El 48,6\% (35 de 72 profesionales) utiliza flujos entre 1 y $5 \mathrm{ml} / \mathrm{h}$, y el 1,4\% (1 de 72 encuestados) utiliza flujos mayores de $5 \mathrm{ml} / \mathrm{h}$.

La jeringa de $10 \mathrm{ml}$ se utiliza por el $65,3 \%$ (47 de 72 profesionales) de los encuestados para la administración de medicamentos en dosis horarias utilizando una BIC; el 55,6\% (40 de 72 profesionales) usa jeringas de $20 \mathrm{ml}$; el 19,4\% (14 de 72 encuestados) utiliza jeringas de $5 \mathrm{ml}$ y el 6,9\% (5 de 72 profesionales) usa jeringas de $50 \mathrm{ml}$, para la misma función.

Las precauciones tomadas por los profesionales al instalar una BIC, que corresponden a medidas que disminuyen los factores que afectan el retraso de inicio de la infusión y el tiempo demorado en alcanzar el flujo deseado, se presentan en la tabla 1 . Sólo uno de los encuestados correspondiente al 1,4\% utiliza siempre un bolo o purgado antes de iniciar una infusión continua; el 18,1\% (13 de 72 profesionales) realiza un bolo en forma ocasional y el $80,6 \%$ (58 de 72 encuestados) de ellos no lo realiza nunca previo a iniciar una infusión. 


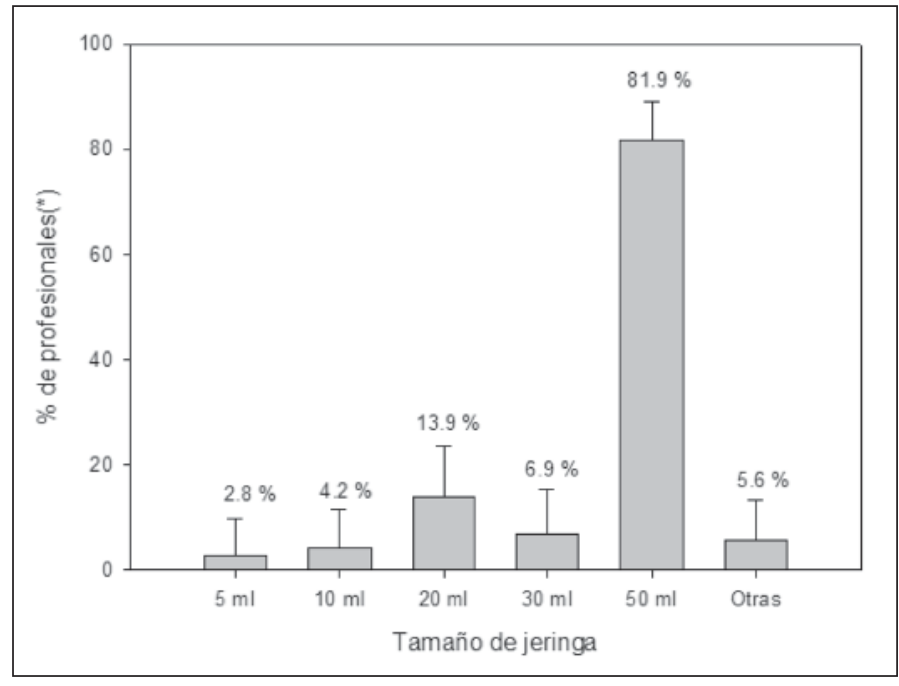

Figura 1. Tamaños de jeringa utilizados para la infusión continua de medicamentos por los profesionales encuestados. *Resultados expresados en porcentaje de profesionales que seleccionan la alternativa como respuesta. La sumatoria de los 6 porcentajes no es igual a $100 \%$ debido a que los profesionales encuestados podían seleccionar más de una alternativa como respuesta.

Tabla 1. Precauciones tomadas por los profesionales encuestados al instalar una bomba de infusión continua

\begin{tabular}{|lc|}
\hline Práctica Clínica & $\begin{array}{c}\text { Resultado } \\
\% \text { (*) }\end{array}$ \\
\hline Eliminación de burbujas del circuito de infusión & $51,4 \%(37 / 72)$ \\
\hline Asegurar conexiones del sistema de infusión & $38,9 \%(28 / 72)$ \\
\hline Adecuado ajuste mecánico entre la jeringa y la bomba de infusión de jeringa & $36,1 \%(26 / 72)$ \\
\hline Cebado del circuito de infusión & $13,9 \%(10 / 72)$ \\
\hline Compatibilidad entre la jeringa y la bomba de infusión de jeringa & $9,7 \%(7 / 72)$ \\
\hline Posición fija de la bomba de infusión de jeringa & $6,9 \%(5 / 72)$ \\
\hline Utilización de un bolo o purgado antes de iniciar el flujo indicado & $1,4 \%(1 / 72)$ \\
\hline
\end{tabular}

(*) En paréntesis números absolutos sobre el total de 72 encuestados.

\section{Discusión}

Los resultados presentados muestran que los profesionales encuestados, pertenecientes a 19 de los principales centros neonatales del país, utilizan con frecuencia flujos menores de $1 \mathrm{ml} / \mathrm{h}$ y jeringas de $50 \mathrm{ml}$ para la infusión continua de medicamentos. Estos resultados son relevantes al considerar el impacto de estas prácticas clínicas en el funcionamiento de las BIC, aumentando significativamente el tiempo demorado en alcanzar un flujo deseado $0^{3,4,7,13}$. Lo anterior cobra importancia clínica particularmente durante la infusión de medicamentos criticos como drogas vasoactivas, insulina, prostaglandina, entre otros, en los que la dosis deseada debe ser lograda rápidamente.

Se han realizado variados estudios que han investigado el uso de flujos bajos y diferentes tamaños de jeringa utilizando distintas marcas de BIC, y los resultados han sido claros. De esta forma se ha demostrado que al utilizar una jeringa de $50 \mathrm{ml}$ y un flujo de $0,4 \mathrm{ml} / \mathrm{h}$ pueden pasar hasta 60 minutos antes del inicio real de la infusión al paciente, y que el flujo deseado no se logra incluso después de 75 minutos de observación ${ }^{13}$. Asimismo, se ha observado que al utilizar flujos menores de $1 \mathrm{ml} / \mathrm{h}$ para la administración de medicamentos en un período de tiempo menor a 60 minutos, el volumen 
efectivamente infundido es menor que el volumen calculado ${ }^{14}$. Esto cobra relevancia durante la infusión de medicamentos en cortos períodos de tiempo ya que se administraría una dosis menor a la deseada. Flujos bajos y jeringas de $50 \mathrm{ml}$ también pueden aumentar el tiempo en que la BIC demora en alcanzar un nuevo flujo. De esta forma si un flujo de $0,1 \mathrm{~m} / \mathrm{h}$ se aumenta a $0,2 \mathrm{ml} / \mathrm{h}$ pueden pasar aproximadamente 15 minutos antes que se alcance la nueva velocidad de infusión del medicamento ${ }^{4}$. La mayoría de estos estudios se han realizado en condiciones experimentales muy controladas, por lo que podemos especular iguales o mayores tiempos de demora de la entrega del medicamento al paciente en las UCIN encuestadas, donde se demostró el uso frecuente de jeringas de $50 \mathrm{ml}$ y flujos menores de $1 \mathrm{ml}$.

Las medidas que permiten disminuir el tiempo de retraso del inicio de una infusión también han sido estudiadas y descritas. Una de ellas es la remoción de burbujas del circuito de infusión. Se han realizado estudios que han demostrado que incluso la presencia de burbujas que impresionan como "clínicamente irrelevantes”, tienen un impacto significativo en el funcionamiento de las $\mathrm{BIC}^{9,10}$. En este estudio se evidenció que aproximadamente sólo el $50 \%$ de los encuestados considera esta medida al instalar una BIC. Asimismo se ha demostrado que los desplazamientos verticales de la BIC durante una infusión generan fluctuaciones importantes en el flujo ${ }^{11,12}$. Sólo el $7,4 \%$ de los profesionales encuestados destaca esta medida al instalar una BIC, por lo que es necesario difundir la importancia de la fijación de la BIC principalmente cuando un paciente requiere ser trasladado. Por otra parte, ha sido demostrado que la administración de un bolo inicial de $2 \mathrm{ml}$ antes de iniciar la infusión y conectarla al paciente, prácticamente elimina el tiempo de retraso y minimiza la demora en la entrega del medicamento ${ }^{7}$. La aplicación de un bolo permite el ajuste preciso del polo posterior del embolo de la jeringa y la BIC, lo cual es fundamental para evitar el espacio libre entre ambos y de esta forma disminuir el tiempo de retraso de la infusión ${ }^{7,8}$. Estas maniobras tampoco se realizan habitualmente en nuestro medio y resultan particularmente importantes al usar BIC que no efectuan un ajuste automatico de la jeringa.

Este estudio presenta algunas limitaciones. La muestra de profesionales no fue realizada al azar, por lo cual no constituye un universo estadísticamente representativo. Por otro lado, en la encuesta se realizó una pregunta abierta de desarrollo para investigar las medidas precautorias que realizan los profesionales al instalar una infusión continua. Esta pregunta puede haber sido ineficiente en demostrar todas las medidas que los profesionales realizan en la práctica clínica en forma rutinaria, ya que muchos de ellos pueden haber olvidado cada uno de los pasos que realizan habitualmente. Sin embargo, creemos que el propósito de esta pregunta fue precisamente investigar cuales medidas son consideradas como relevantes por los encuestados.

Este es el primer estudio que ha cuantificado en nuestro medio la utilización de las principales medidas que impactan sobre el retraso de tiempo de inicio de la infusión, y ha evidenciado que estas precauciones no son realizadas rutinariamente por los profesionales encuestados. Considerando el impacto negativo de esta práctica clínica en la adecuada infusión de medicamentos a los pacientes, es fundamental generar un cambio eficaz de conducta en esta área. Por lo tanto, proponemos una guía de recomendaciones basadas en la literatura

Tabla 2. Recomendaciones prácticas para el uso de bombas de infusión continua de jeringa

1. Preferir flujos $>1 \mathrm{ml} / \mathrm{h}$ para la infusión continua de medicamentos

2. Al usar flujos $\leq 1 \mathrm{ml} / \mathrm{h}$ para la infusion continua de medicamentos recomendamos utilizar la jeringa de menor tamaño posible evitando el uso de jeringas de $50 \mathrm{ml}$

3. Al administrar medicamentos en bolo preferir flujos $>1$ $\mathrm{ml} / \mathrm{h}$ y tiempos de infusion $\geq 1 \mathrm{~h}$

4. Minimizar el espacio libre entre el émbolo de la jeringa y la bomba antes de iniciar una infusion de medicamentos

5. Minimizar las burbujas en la jeringa y circuito antes de iniciar la infusión

6. Realizar un bolo o purgado de $2 \mathrm{ml}$ antes de conectar circuito de infusión al paciente

7. Evitar desplazamientos de la BIC en el eje vertical durante la infusión 
existente que minimizan los problemas previamente detallados y que se exponen en la tabla 2. Es relevante difundir estas simples medidas en las UCIN de Chile ya que tienen un impacto significativo en el cuidado intensivo neonatal.

\section{Agradecimientos}

Iris Delgado, Clínica Alemana de Santiago, Universidad del Desarrollo.

Centros participantes: Clínica Alemana de Santiago, Clínica Dávila, Clínica Indisa, Hospital Barros Luco Trudeau, Hospital Carlos Van Buren, Hospital Clínico de la Pontificia Universidad Católica de Chile, Hospital Clínico de la Universidad de Chile José Joaquín Aguirre, Hospital de La Serena, Hospital de Osorno, Hospital de Puerto Montt, Hospital Dr. Gustavo Fricke, Hospital Dr. Sótero del Río, Hospital Luis Calvo Mackenna, Hospital Santiago Oriente Dr. Luis Tisné Brousse, Hospital Padre Hurtado, Hospital Parroquial de San Bernardo, Hospital San Borja Arriarán, Hospital San José, Hospital San Juan de Dios.

\section{Referencias}

1.- Rooke GA, Bowdle TA: Syringe pumps for infusion of vasoactive drugs: mechanical idiosyncrasies and recommended operating procedures. Anesth Analg 1994; 78: 150-6.

2.- Dunster KR, Colditz PB: Flow continuity of infusion systems at low flow rates. Anaesth Intens Care 1995; 23: 605-9.

3.- Weiss M, Hug MI, Neff T, Fischer J: Syringe size and flow rate affect drug delivery from syringe pumps. Can J Anaesth 2000; 47: 1031-5.

4.- Neff SB, Neff TA, Gerber S, Weiss MM: Flow rate, syringe size and architecture are critical to start-up performance of syringe pumps. Eur J Anaesthesiol 2007; 24: 602-8.

5.- Weiss M, Banziger O, Neff F, Fanconi S: Influence of infusion line compliance on drug delivery rate during acute line loop formation. Intensive Care Med 2000; 26: 776-9.

6.- Weiss M, Neff T, Gerber A, Fischer J: Impact of infusion line compliance on syringe pump performance. Paediatr Anaesth 2000; 10: 595-9.

7.- Neff T, Fischer J, Fehr S, Baenziger O, Weiss M: Startup delays of infusion syringe pumps. Paediatr Anaesth 2001; 11: 561-5.

8.- Lonnqvist PA, Lofqvist B: Design flaw can convert commercially available continuous syringe pumps to intermittent bolus injectors. Intensive Care Med 1997; 23: 998-1001.

9.- Schulz G, Fischer J, Neff T, Banziger O, Weiss M: The effect of air within the infusion syringe on drug delivery of syringe pump infusion systems. Anaesthesist 2000; 49: 1018-23.

10.- Davey C, Stather-Dunn T: Very small air bubbles (10-70 microl) cause clinically significant variability in syringe pump fluid delivery. J Med Eng Technol 2005; 29: 1306.

11.- Neff TA, Fischer JE, Schulz G, Baenziger O, Weiss M: Infusion pump performance with vertical displacement: effect of syringe pump and assembly type. Intensive Care Med 2001; 27: 287-91.

12.- Kern H, Kuring A, Redlich U, et al: Downward movement of syringe pumps reduces syringe output. Br J Anaesth 2001; 86: 828-31.

13.- Schmidt N, Sáez C, Seri I, Maturana A: Impact of syringe size on the performance of infusion pumps at low flow rates. Ped Crit Care Med 2010; 11: 282-6.

14.- Aubel F, Bernstein T, Fuentes C, et al: Variations in effective volume delivery using a continuous infusion syringe pump at low flow infusion rates. E-PAS 2006: 59: 4850-217. 